\title{
RÉSULTATS PRÉCOCES D'ESSAIS \\ DE FERTILISATION DU PEUPLIER SUR SOLS A GLEY DANS LA MEUSE
}

\author{
Ph. LEROY \\ avec la collaboration technique de G. Courrier \\ Station de Recherches sur les Sols forestiers et la Fertilisation \\ Centre national de Recherches forestières, 54 - Nancy \\ Institut national de la Recherche agronomique
}

\begin{abstract}
SOMMAIRE
Une série d'essais permet de proposer une formule de fertilisation susceptible de réduire les effets néfastes du Dothichiza populea et d'améliorer la croissance sur des jeunes plantations de peupliers installées sur des sols à gley calciques de l'est de la France. En outre, laction dépressive de la concurrence herbacée a été mise en évidence; la destruction des adventices par fauchages ou herbicides serait bénéfique.

La souplesse des dispositifs expérimentaux utilisés et un contrôle rigoureux ont permis d'aboutir à ces résultats deux ans après la mise en place des essais.
\end{abstract}

\section{I. - INTRODUCTION}

Les essais de fertilisation restent une des méthodes d'étude privilégiées des relations entre la nutrition minérale et la croissance des peupliers. Cependant, les résultats actuellement utilisables forment un ensemble trop hétérogène poür conduire à des conclusions de portée générale. Chacun des essais semble en effet constituer un cas particulier, sans qu'il soit possible d'établir entre eux de relations à caractère synthétique. Il est probable que ces relations apparaîtraient si on multipliait le nombre des plantations expérimentales, en tenant compte de leur situation écologique. C'est là l'intérêt que pourraient avoir des réseaux d'essais de fertilisation installés sur chacun des types de sols à peuplier (1). L'ensemble des résultats obtenus à partir de ces réseaux permettrait :

a) de contrôler et de compléter les connaissances acquises sur la croissance du peuplier par d'autres méthodes d'investigation ;

(1) Pour les résineux, ces réseaux tendent à prendre actuellement un développement intéressant, notamment celui du Massif Central créé par M. Bonneau. 
b) de fournir directement aux populiculteurs des indications de portée pratique, vérifiées expérimentalement.

C'est dans ce contexte que se poursuivent les créations d'essais de fertilisation sur Peuplier. La station «Sols forestiers»du C.N.R.F. contribue à cet effort dans la me;ure de ses moyens. En 1966, furent ainsi lancés les essais dont nous commentons ici les résultats. Il s'agit d'essais sur sols à gley calcaires développés sur alluvions de la Meuse (sols de marais). Ce type de sols, fréquemment rencontré, a la populiculture pour vocation traditionnelle. Les quelques essais de fertilisation existant en France sur des stations de même nature aboutissent à des résultats très partiels et même contradictoires que nous voulions compléter. Ainsi pour Chardenon (1957), les formules phospho-potassiques se sont souvent avérées les plus favorables, tandis que pour ConTANT (1957), les formules à dominance azotée se sont montrées très efficaces. De telles imprécisions pourraient d'ailleurs être relevées à propos de tous les types de sols à peuplier et particulièrement pour les sols alluviaux qui ont essentiellement intéressé les expérimentateurs.

Sur un tout autre plan, on reproche souvent aux essais de fertilisation de nécessiter la mise en place de dispositifs coûteux et de ne conduire à des résultats utilisables qu'après de longs délais. Nous verrons que ces inconvénients peuvent être largement atténués par le choix de méthodes expérimentales bien adaptées.

Notons enfin que, sur le plan de l'économie régionale, les essais sur sols à giey de la Meuse à texture argileuse offraient un certain caractère d'urgence. De nombreuses peupleraies installées sur ces sols sont, en effet, dans un tel état de faiblesse quelques années après la plantation, qu'elles risquent de disparaître, en dépit des investissements élevés, notamment drainage, qu'elles ont exigés.

\section{II. - MATERIEL ET METHODES}

\section{1. - Caractéristiques de la station}

\subsection{Situation et passé cultural.}

Les plantations ont été réalisées en forêt domaniale du Morthomme à $15 \mathrm{~km}$ au nordouest de Verdun, sur des alluvions de la Meuse, inondées par les crues d'hiver et dans lesquelles le plan d'eau avant drainage ne descendait pas en dessous de $40 \mathrm{~cm}$. Avant les essais, les terrains utilisés ont porté une peupleraie plantée en 1930 et exploitée en 1959 (clône inconnu) : le rendement moyen ne fut que de $6,6 \mathrm{~m} \% / \mathrm{ha} /$ an pour 250 plants/ha. Les arbres étaient chétifs et ont été abattus à la suite d'une attaque de Dothichiza populea (1).

\subsection{Description et analyses de sol.}

Les essais sont installés sur des sols à anmoor calcique (sols humiques à gley, DuchauFour, 1965) qui présentent une grande homogénéité morphologique.

On peut en donner la description suivante : et plastique.

- $A_{1}: 20$ à $30 \mathrm{~cm}$. Anmoor calcique brun noir à structure fondue ; consistance grasse

$-\mathrm{G}_{0}: 0$ à $10 \mathrm{~cm}$. Concrétions ferriques sur fond gris. Structure fondue.

$-\mathrm{G}_{\mathrm{R}}$ : structure fondue et teinte uniforme gris-verdâtre.

(1) Dothichiza populea SACC. et BrIARD. 
- Niveau estival de la nappe : 30 à $40 \mathrm{~cm}$.

- Débris de coquilles calcaires sur tout le profil.

En été 1965, des travaux de drainage ont abaissé le niveau estival de la nappe à 60 ou $70 \mathrm{~cm}$, mais en 1968 aucune modification visible du profil n'était encore apparue en dehors de l'effet d'assainissement des horizons supérieurs.

Les analyses mettent en évidence une certaine hétérogénéité des caractéristiaues des sols; cette hétérogénéité est normale sur alluvions. Au tableau 1 figurent deux analyses qui donnent l'image de la fourchette de variation pour les terrains en expérience. On peut, en fait, grouper l'ensemble des sols par les points suivants: texture argileuse, structure massive, présence de calcaire actif, abondance de matière organique à $\mathrm{C} / \mathrm{N}$ bas, richesse relative en bases échangeables, teneur moyenne en $\mathrm{P}_{2} \mathrm{O}_{5}$.

TABLEAU 1

Résultats et méthodes d'analyses des sols

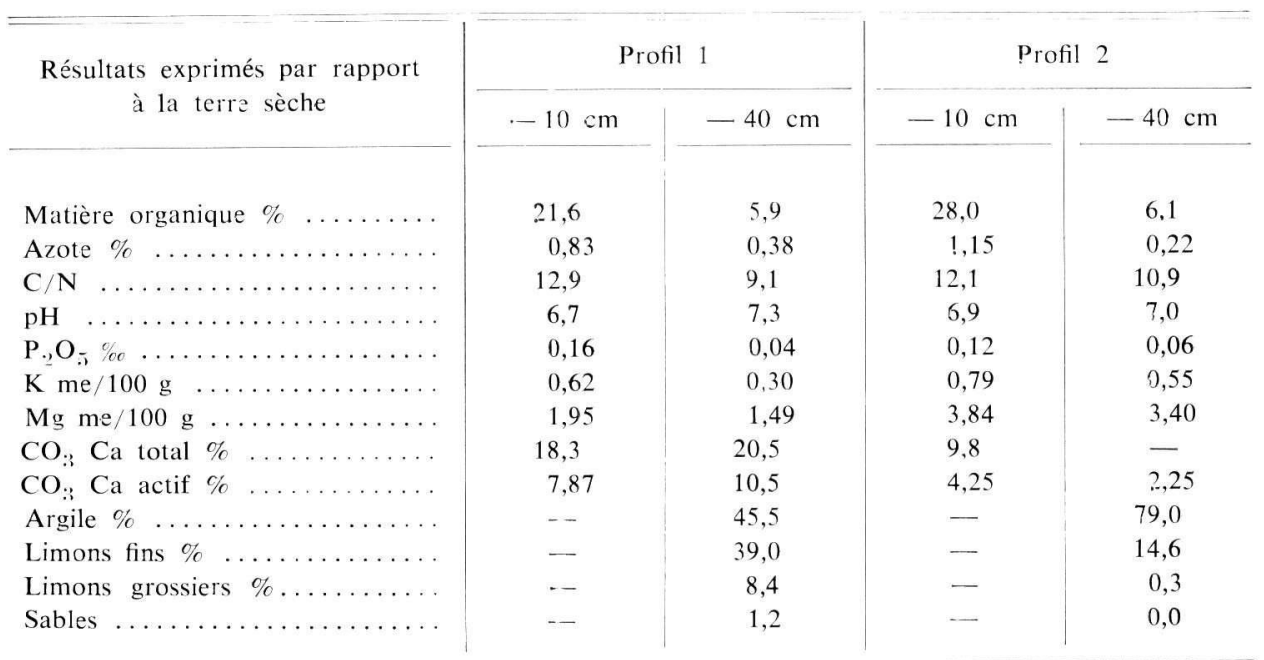

Matière organique : dosage du carbone par la méthode Anne.

Azote total: méthode Kjeldahl.

$\mathrm{pH}: \mathrm{pH}$ à l'eau.

Phosphore assimilable : méthode Duchaufour. Double extraction : $\mathrm{SO}_{4} \mathrm{H} ., \mathrm{N} / 250+\mathrm{NaOH} \mathrm{N} / 10$.

$\mathrm{K}$ et $\mathbf{M g}$ échangeables : percolation acétate d'ammonium.

Calcaire total: calcimètre Bernard.

Calcaire actif : méthode Drouineau.

Granulométrie: méthode Mériaux par densimétrie, sans destruction du calcaire.

Sur ces sols de marais, la végétation naturelle est très abondante. Dans les zones d'essai, dominent les Carex en mélange avec la Spirée et la Valériane.

\section{2. - Matériel végétal et modalités de plantation}

Les plantations expérimentales ont toutes été réalisées avec $P$. euramericana $s$. Tardif de Champagne. Dans les conditions de l'essai, on peut en attendre une production de 8 à $10 \mathrm{~m}^{3} / \mathrm{ha} / \mathrm{an}$ en l'absence de fertilisation.

Les plantations ont été effectuées en novembre 1966 et novembre 1967, en respectant les normes suivantes:

- plants racinés. Circonférence moyenne de 8 à $9 \mathrm{~cm}$;

- distances de plantation: $7 \times 7 \mathrm{~m}$; 
- trous de plantation: $50 \times 50 \times 60 \mathrm{~cm}$. Aucun travail du sol n'a pu être réalisé sur les parcelles d'essai à cause de la trop faible portance des terrains.

\section{3. - Doses et modes d'apport des engrais}

Trois éléments N, P, K, ont été appliqués seuls ou en combinaisons. Pour chacun d'eux, des doses et des modes d'apport standardisés ont été adoptés :

Phosphore $(P)$ :

dose : $150 \mathrm{~g} \quad \mathrm{P}_{2} \mathrm{O}_{5} /$ plant sous forme de superphosphate triple à $45 \%$, soit environ $1 \mathrm{~g}$ de $\mathrm{P}_{2} \mathrm{O}_{5}$ par $\mathrm{dm}^{3}$ de potet ;

apport: en une fois au trou de plantation, en mélange avec la terre de remplissage, en prenant la précaution d'isoler les racines par une couche de terre non enrichie.

Potassium $(K)$ :

dose: $75 \mathrm{~g} \mathrm{K.2}$ /plant sous forme de sulfate de potassium, soit environ $0,5 \mathrm{~g}$ de $\mathrm{K}_{2} \mathrm{O}$ par $\mathrm{dm}^{3}$ de potet ;

apport: idem à $\mathrm{P}$.

Azote $(N)$ :

doses: juin $1^{\text {re }}$ année : $60 \mathrm{~g} \mathrm{~N} /$ plant,

juin $2^{\mathrm{e}}$ année : $60 \mathrm{~g} \mathrm{~N} /$ plant ;

apports répétés en surface sous forme d'ammonitrate à $34.5 \%$,

$1^{\text {re }}$ année : épandage sur un rayon de $50 \mathrm{~cm}$ autour du pied;

$2^{\circ}$ année: rayon de $70 \mathrm{~cm}$.

Dans la suite du texte, les traitements comportant un ou plusieurs cies symboles N, P ou $\mathrm{K}$, auront reçu des engrais suivant des modalités correspondant à la liste établie ci-dessus. Le symbole $\mathrm{T}$ indique le traitement témoin ne comportant aucune fertilisation.

\subsection{Mensurations.}

\section{4. - Contrôle des essais}

Elles ont été réalisées en novembre des années 1966, 1967 et 1968. Elles ont porté sur les circonférences à $1,30 \mathrm{~m}$ du collet de 1966 à 1968. Les hauteurs ont en outre été mesurées en 1966 et 1967.

\subsection{Contrôle foliaire.}

Ce contrôle a été réalisé à partir d'échantillonnages portant sur des feuilles bien développées, cueillies sur des rameaux de la partie supérieure des cimes en début août 1967 et 1968 (VAN Der MEIDEN, 1964).

Chaque échantillon regroupait des feuilles prélevées sur un minimum de 10 arbres ayant subi les mêmes traitements.

Les feuilles ont fait l'objet de mensurations et de pesées. Les analyses ont porté sur $\mathrm{N}, \mathrm{P}, \mathrm{K}, \mathrm{Ca}, \mathrm{Mg}$ en suivant les méthodes classiques (erreurs analytiquies respectives au seuil de $5 \%: 2 \%-0 \%-1 \%-2 \%-10 \%)$.

L'interprétation statistique des divers résultats d'analyse foliaire a permis d'établir que les plus petites différences significatives $(5 \%)$ pour $\mathrm{N}, \mathrm{P}$ et $\mathrm{K}$ étaient respectivement de $0,52-0,10$ et $0,50 \%$ en valeur absolue. Pour $\mathrm{Ca}$ et $\mathrm{Mg}$, aucune différence statistiquement vérifiée n'est apparue. Il aurait été possible d'abaisser ces seuils de signification en multipliant les analyses: cela est difficilement réalisable sur le plan matériel dans le cadre d'essais de fertilisation.

Dans ce cas présent, nous pensons que des écarts inférieurs à ceux que nous avons calculés ont une valeur réelle, d'autant plus que nous travaillons sur un clone où la variabilité génétique est nulle.

\section{III. - RESULTATS DES ESSAIS PLANTES EN 1966}

En novembre 1966, deux dispositifs furent installés sur une partie de la surface disponible (1). Le dispositif principal repose sur la méthode des blocs : 5 traitements,

(1) Voir modalités de plantation et de fertilisation au chapitre II, $\$ 2.2$. et 2.3 . 
4 répétitions, 25 plants par placeau, soit 500 plants en expérience. Un essai mono-arbre annexe complète ce premier dispositif; il groupe factoriellement les trois éléments $\mathrm{N}$, $\mathrm{P}$ et $\mathrm{K}: 8$ traitements, 25 répétitions, soit 200 plants en expérience.

\section{1. - Dispositif principa!}

Les cinq traitements T - NP - NK - PK - NPK ont fait l'objet d'un contrôle par mensurations et analyses foliaires.

\subsection{1. - Résultats des mensurations}

Les mesures ont porté sur 9 plants par placeau; au tableau 2 apparaissent les circonférences moyennes observées en 1966, 1967 et 1968. Les traitements T et PK se distinguent nettement du groupe des traitements comportant de l'azote, sans qu'il soit réellement possible de procéder à une analyse plus fine.

\section{TABLEAU 2}

Dispositif principal. Circonférences moyennes des plants en $\mathrm{mm}$ (mesure à $1,30 \mathrm{~m}$ du sol)

\begin{tabular}{|c|c|c|c|c|c|}
\hline Date des mesures & $\mathrm{T}$ & NP & NK & PK & NPK \\
\hline Novembre $1966 \ldots$ & 76,38 & 77,77 & 79,72 & 77,77 & 74,72 \\
\hline Novembre $1967 \ldots$ & 80,88 & 83,47 & 86,82 & 81,57 & 83,52 \\
\hline Novembre $1968 \ldots$ & 94,56 & 107,26 & 107,18 & 91,46 & 107,34 \\
\hline
\end{tabular}

Interprétation statistique par test de Duncan (Millier, 1967).

1966 : aucune différence significative.

1967 : NK significativement différent de T et PK.

1968 : NK, NP, NPK significativement différents de T et PK.

Le tableau 3 présente les accroissements annuels moyens déduits des mesures de circonférence. Par ce biais, l'influence des différences de départ entre traitements est éliminée et les effets des divers apports d'engrais apparaissent plus nettement. Ainsi, en 1967, les traitements se classaient dans l'ordre d'effet croissant suivant (1) :

$\begin{array}{llll}\text { PK } & \mathrm{T} & \mathrm{NP} & \mathrm{NK}\end{array}$

L'extrême faiblesse des accroissements 1967 illustre parfaitement les difficultés d'installation des plants sur la station.

En 1968, on retrouve un classement presque identique :

$\begin{array}{llll}\text { PK } & \mathrm{T} & \mathrm{NK} & \mathrm{NP} \\ \end{array}$

(1) Un trait continu sous un ensemble de traitements signifie qu'il n'y a pas de différence significative entre les traitements de cet ensemble. 
Ainsi, en deuxième année de végétation, les plants ayant reçu une fertilisation azotée ont des accroissements normaux (normale estimée à $20 \mathrm{~mm}$ pour le clone employé). Au contraire, les plants témoins ou fertilisés PK se distinguent encore nettement par la faiblesse de leur croissance.

\section{TABLEAU 3}

Dispositif principal. Accroissements annuels moyens sur la circonférence en $\mathrm{mm}$ (mesure à $1,30 \mathrm{~m}$ du sol)

\begin{tabular}{|c|c|c|c|c|c|c|c|}
\hline Accroissemen & $t$ annuel & $\mathrm{T}$ & NP & NK & PK & NPK & p.p.d.s. \\
\hline Accroissement & 1967 & 4,5 & 5,7 & 7,1 & 3,8 & 8,8 & - \\
\hline$\gg$ & $1968 \ldots$ & 13,68 & 23,79 & 20,36 & 9,89 & 23,82 & 9,33 \\
\hline$\gg$ & total .. & 18,18 & 29,49 & 27,46 & 13,69 & 32,62 & 11,90 \\
\hline
\end{tabular}

p.p.d.s. : plus petite différence significative à $5 \%$.

Interprétation statistique :

- accroissement 1967: test de Duncan. NPK diffère significativement de PK.

- accroissement 1968: analyse en blocs.

- accroissement total : analyse en blocs.

Le classement général des accroissements cumulés 1967-1968 présente enfin le schéma suivant :

$\begin{array}{llll}\text { PK } & \mathrm{T} & \text { NK } & \text { NP }\end{array}$

Les commentaires des tableaux 2 et 3 permettent en définitive de constater que :

- les formules de fertilisation contenant de l'azote ont des effets significatifs sur la croissance. Le traitement NK serait le moins efficace,

- la fertilisation phospho-potassique seule n'a pas d'effet et pourrait même avoir un effet dépressif.

Des mesures réalisées sur les hauteurs en 1966 et 1967 complètent ces observations en confirmant les difficultés d'installation des plants:

- hauteur moyenne de départ: $3,9 \mathrm{~m}$,

— pousse en hauteur moyenne de $7,6 \mathrm{~cm}$ pour les plants ayant reçu de l'azote ; pousse nulle pour les autres.

Sur un plan différent, des calculs de corrélation montrent que les accroissements de départ semblent être d'autant plus faibles que la circonférence initiale est plus forte, sauf pour le traitement NPK le plus favorable à la croissance où l'on ne relève aucune corrélation significative.

\subsection{2. - Mensurations et analyses de feuilles.}

Le tableau 4 présente les résultats de pesées et mensurations de feuilles qui confirment les observations sur la croissance en circonférence; le classement en fonction de ces caractères est en effet du type :

$$
\text { T PK NP NK NPK }
$$


Le traitement NPK est toujours significativement différent des traitements $T$ et PK et va jusqu'à doubler le poids sec de 1.000 feuilles. Au niveau foliaire on constate en 1968 une tendance à la supériorité du traitement NK sur le traitement NP, alors qu'on observait le contraire à propos des accroissements en circonférence.

TABLEAU 4

Dispositif principal. Poids des feuilles et dimensions des limbes

\begin{tabular}{|c|c|c|c|c|c|c|}
\hline & $\mathrm{T}$ & NP & NK & PK & NPK & p.p.d.s. \\
\hline Ps 1967 (1) ........ & 144 & 254 & 246 & 118 & 275 & 84 \\
\hline Ps 1968 (1) $\ldots \ldots \ldots$ & 260 & 367 & 403 & 310 & 578 & 84 \\
\hline $1 \quad 1968$ (2) $\ldots \ldots \ldots$ & 7,23 & 8,26 & 8,96 & 7,81 & 9,76 & 1,4 \\
\hline L 1968 (3) $\ldots \ldots \ldots$ & 7,76 & 8,76 & 9,62 & 8,19 & 9,94 & 1,4 \\
\hline
\end{tabular}

(1) Ps: Poids sec de 1.000 feuilles en g.

(2) 1: Largeur des limbes en $\mathrm{cm}$.

(3) L: Longueur des limbes en $\mathrm{cm}$.

Les tableaux 5 et 6 regroupent les résultats d'analyses foliaires (cf. chap. II paragraphe 2.42). La comparaison des teneurs en N, P et K pour 1967 et 1968 met en évidence les difficultés de nutrition rencontrées par les plants au cours de leur première saison de végétation et ceci quelle que soit la formule de fertilisation. Ces difficultés sont très bien illustrées au tableau 6 par les écarts entre poids d'éléments contenus dans 1.000 feuilles des deux premières années de végétation.

Les plants témoins montrent d'ailleurs en 1967 des taux foliaires en $\mathrm{N}$ et $\mathrm{K}$ inférieurs aux seuils de carence admis chez le peuplier. Le cas du calcium est particulier: les teneurs en cet élément sont, en effet, plus fortes en 1967 qu'en 1968 et même en 1968 c'est le témoin qui montre le taux le plus élevé.

L'effet des traitements se manifeste nettement. En 1967, les carences foliaires en un élément disparaissent quand celui-ci est apporté par la fumure. Le traitement NPK se montre ainsi nettement favorable. Les traitements NP et NK s'avèrent, par contre, déséquilibrés; ils ont respectivement tendance à diminuer les teneurs foliaires en $\mathrm{K}$ ou $\mathrm{P}_{2} \mathrm{O}_{\overline{5}}$. En ce domaine, la fertilisation NP semble plus dangereuse puisqu'elle induit une carence nette en potassium. Ces déséquilibres doivent être accentués par des phénomènes de dilution ; les traitements NP et NK ont, en effet, une influence positive sur la fabrication de matière sèche foliaire (tableau 4). Le traitement $P K$ enfin s'avère plutôt défavorable: il tend à diminuer le taux d'azote foliaire.

Les résultats 1968 conduisent à des commentaires de même nature, en notant cependant que les carences ne se manifestent généralement plus en seconde année. Le traitement $\mathrm{PK}$ confirme son caractère dépressif sur la nutrition en provoquant à la fois une carence azotée liée à un effet de dilution, et semble-t-il une consommation de luxe en potassium. 
TABLEAU 5

Dispositif principal. Analyses foliaires

(lıs résultats sont indiqués en $\%$ de la matière sèche)

\begin{tabular}{|c|c|c|c|c|c|}
\hline & $N$ & $\mathrm{P}_{2} \mathrm{O}_{5}$ & K & $\mathrm{Ca}$ & $\mathrm{Mg}$ \\
\hline \multicolumn{6}{|l|}{ Août 1967 : } \\
\hline $\mathbf{T} \ldots \ldots \ldots$ & 1,89 & 0,46 & 1.09 & 2,72 & 0,27 \\
\hline NP $\ldots \ldots \ldots$ & 2,15 & 0,51 & 0,87 & 2,94 & 0,23 \\
\hline NK $\ldots \ldots \ldots$ & 2,30 & 0,40 & 1.63 & 2,78 & 0,13 \\
\hline PK $\ldots \ldots \ldots$ & 1,75 & 0,80 & 1,48 & 3,52 & 0,18 \\
\hline NPK $\ldots \ldots$ & 2,51 & 0,56 & 1,66 & 2,56 & - \\
\hline Moyenne $1967 \ldots$ & 2,12 & 0,55 & 1,35 & 2,90 & 0,20 \\
\hline \multicolumn{6}{|l|}{ Août 1968 : } \\
\hline $\mathrm{T} \ldots \ldots \ldots$ & 2,41 & 0,68 & 1,62 & 2,34 & 0,19 \\
\hline NP $\ldots \ldots \ldots$ & 2,88 & 0,62 & 1,35 & 2,19 & 0,22 \\
\hline NK $\ldots \ldots \ldots$ & 2,88 & 0,63 & 1,40 & 2,30 & 0,20 \\
\hline PK $\ldots \ldots \ldots$ & 2,07 & 0,68 & 1,97 & 2,06 & 0,11 \\
\hline NPK $\ldots \ldots$ & 2,77 & 0,53 & 1,31 & 2,25 & 0,21 \\
\hline Moyenne $1968 \ldots$. & 2,60 & 0,63 & 1,53 & 2,23 & 0,19 \\
\hline Seuils de carence. & 2,2 & 0,40 & 1,30 & - & 0,15 \\
\hline
\end{tabular}

Interprétation statistique : cf. chap. II $\$ 242$.

TABLEAU 6

Dispositif principal. Poids d'éléments minéraux contenus dans 1000 feuilles sèches (g)

\begin{tabular}{|c|c|c|c|c|c|}
\hline & $\mathrm{N}$ & $\mathrm{P}_{2} \mathrm{O}_{5}$ & K & $\mathrm{Ca}$ & $\mathrm{Mg}$ \\
\hline \multicolumn{6}{|l|}{ Août 1967: } \\
\hline T $\ldots \ldots$ & 2,72 & 0,66 & $!, 57$ & 3,92 & 0,39 \\
\hline$N P \ldots \ldots \ldots$ & 5,46 & 1,29 & 2,21 & 7,47 & 0,58 \\
\hline NK $\ldots \ldots \ldots$ & 5,66 & 0,98 & 4,01 & 6,84 & 0,32 \\
\hline PK $\ldots \ldots$ & 2,06 & 0,94 & 1,75 & 4,15 & 0,21 \\
\hline NPK $\ldots \ldots$ & 6,90 & 1,54 & 4,56 & 7,04 & - \\
\hline Moyenne $1967 \ldots$. & 4,56 & 1,08 & 2,82 & 5,88 & 0,38 \\
\hline Août 1968 : & & & & & \\
\hline $\mathrm{T} \ldots \ldots$ & 6,27 & 1,77 & 4,21 & 6,08 & 0,49 \\
\hline NP $\ldots \ldots \ldots$ & 10,57 & 2,28 & 4,95 & 8,04 & 0,81 \\
\hline NK $\ldots \ldots \ldots$ & 11,61 & 2,54 & 5,64 & 9,27 & 0,81 \\
\hline PK $\ldots \ldots \ldots$ & 6,42 & 2,11 & 6,11 & 6,38 & 0,34 \\
\hline NPK $\ldots \ldots$ & 16,01 & 3,06 & 7,57 & 13,00 & 1,21 \\
\hline Moyenne $1968 \ldots$. & 10,18 & 2,35 & 5,70 & 8,55 & 0,73 \\
\hline
\end{tabular}


En définitive, deux idées générales se dégagent de cet ensemble de résultats:

- le traitement NPK est de loin le plus favorable à la nutrition,

- les observations sur la croissance et les commentaires d'analyse foliaire semblent en étroite concordance.

\section{2. - Essai monoarbre}

Cet essai avait pour but de compléter les résultats obtenus sur le dispositif principal. Ce type d'essai permet, en effet, de tester un grand nombre de traitements sur une surface restreinte. En fait, chaque plant constitue un placeau élémentaire et un grand nombre de répétitions assure la rigueur de l'interprétation. Dans le cas présent. 8 traitements sont répétés 25 fois. Ce mode d'expérimentation est bien adapté au peuplier où la variabilité génétique sur un clone est nulle. Les essais «monoarbre» présentent, cependant, l'inconvénient de ne fournir des résultats valables que pendant les quelques années où les racines de deux plants voisins subissant des traitements différents ne se rejoignent pas.

Les résultats de l'essai monoarbre confirment, en les nuançant, les conclusions déjà apportées (cf. tableau 7).

\section{TABLEAU 7}

Essai monoarbre. Accroissements annuels moyens sur la circonférence en $\mathrm{mm}$ (mesure à $1,30 \mathrm{~m}$ du sol)

\begin{tabular}{|c|c|c|c|c|c|c|c|c|c|c|}
\hline & & $\mathrm{T}$ & $\mathrm{N}$ & $P$ & K & $\mathrm{NP}$ & NK & PK & NPK. & p.p.d.s. \\
\hline Accroiss & 1967 & 3,8 & 6,5 & 3,2 & 4,1 & 6.5 & 4,3 & 4,1 & 8,3 & 4,1 \\
\hline$\gg$ & 1968 & 6,5 & 14,0 & 8,5 & 11,8 & 19,9 & 28,9 & 15,9 & 24,8 & 6,0 \\
\hline$\gg$ & total & 10,3 & 20,5 & 11,7 & 15,9 & 26,4 & 33,2 & 20,0 & 33,1 & 8,6 \\
\hline
\end{tabular}

p.p.d.s. : plus petite différence significative au seuil de $5 \%$,

Les classements des accroissements moyens en circonférence en fonction des divers traitements, peuvent, en effet, se schématiser ainsi :

accroissement 1967: P - T - (K - PK) - NK - (NP - N) - NPK

accroissement 1968: T - P - K - N - PK - NP - NPK - NK

accroissement total : $T-P-\bar{K}-P K-N-\overline{N P}-(N P K-N K)$

Ici encore, les formules de fertilisation contenant de l'azote ont les effets les plus significatifs sur la croissance. L'azote seul est plus efficace que le phosphore ou le potassium employés séparément.

Cependant, à la différence du dispositif principal, la fumure PK n'apparaît pas avec un caractère dépressif et d'autre part, les formules NK et NPK semblent équivalentes. 


\section{3. - Effet des engrais sur le Dothichiza}

De nombreux plants en expérience ont été atteints par le Dothichiza. Ce champignon, parasite de faiblesse, s'attaque aux écorces et désorganise les tissus sous-jacents. Il peut provoquer la mort de l'arbre par décortication annulaire. Couramment rencontré dans l'est de la France, il cause des dégâts importants aux jeunes plantations de peupliers en crise d'installation, qui ne peuvent cicatriser leurs plaies par une croissance rapide. Lorsque cette crise risque d'être sévère, le moyen de lutte le plus efficace contre le Dothichiza est encore d'effectuer les plantations au printemps afin que les plants n'aient pas à traverser leur premier hiver en état de faiblesse; c'est, en effet, essentiellement au cours de cette saison que le champignon se propage (TARIS, 1957). Malheureusement, la nécessité d'étaler les travaux dans le temps empêche souvent de procéder de cette façon.

Les peupliers en expérience, plantés en novembre, se trouvaient donc placés au départ dans les conditions les plus difficiles, et la mortalité due au Dothichiza s'est élevée aux environs de $30 \%$ fin 1968 ; cette mortalité ne fut cependant pas de même intensité selon les traitements. On peut, en effet, relever les pourcentages suivants :

$\begin{array}{cccccccc}38 \% & 21 \% & 22 \% & 37 \% & 36 \% & 18 \% & 47 \% & 13 \% \\ \mathrm{~T} & \mathrm{~N} & \mathrm{P} & \mathrm{K} & \mathrm{NP} & \mathrm{NK} & \mathrm{PK} & \mathrm{NPK}\end{array}$

Le traitement NPK a diminué le taux de mortalité de façon significative et cette action positive doit être rapprochée de ses effets sur la croissance (1). Au contraire, la formule PK favorise la virulence du parasite. Il faut, en outre, noter que le traitement NK a diminué la mortalité d'environ moitié par rapport au témoin et aux formules NP et $\mathrm{K}$.

La supériorité de l'association NPK apparaît nettement, puisqu'elle joue à la fois sur la croissance et la résistance au Dothichiza. Mais c'est peut-être sur ce dernier point que la fumure NPK s'avérera la plus rentable à court terme. Elle permet, en effet, dans l'exemple présent de faire passer le nombre des regarnis d'environ 76 à 26 plants/ha, soit une économie de l'ordre de 350 à $400 \mathrm{~F}$ par hectare dès la deuxième année de plantation, pour un investissement engrais de 50 à $100 \mathrm{~F}$ par hectare.

\section{IV. - DISCUSSION DES RESUltatS}

Les résultats des essais 1966 permettent de tirer un certain nombre de conclusions :

- les traitements contenant de l'azote tendent à favoriser la croissance. La fumure NPK est de loin la plus favorable, tant sur le plan de la nutrition que sur celui de la croissance (gain min. de $77 \%$ sur les accroissements en circonférence),

- les formules binaires NP et NK, tout en pouvant agir favorablement sur la croissance, entraînent des déséquilibres nutritionnels (particulièrement marqués pour la fumure NP),

(1) De nombreuses mensurations ont montré que le Dothichiza n’était plus à craindre quand l'accroissement dépassait $15 \mathrm{~mm}$ (POURTET, communication personnelle). Les résultats d'essai confirment cette observation, cenendant l'état d'infection par le Dothichiza est tel qu'un certain degré de mortalité sur les placettes fertilisées risque encore de se manifester. 
- le traitement PK n'a que peu d'effet sur la croissance et pourrait même avoir un effet dépressif. Il conduit, par ailleurs, à un déséquilibre nutritionnel marqué,

- la résistance au Dothichiza est d'autant plus forte que la croissance est meilleure. Le traitement NPK fait ainsi passer la mortalité de 38 à $13 \%$. Le traitement PK, au contraire, accentue les dégâts.

En définitive, sur la station d'essai à texture argileuse et plan d'eau proche de la surface, les plants souffrent au départ de difficultés de nutrition minérale (1) qui se traduisent par une croissance extrêmement faible et une grande sensibilité aux parasites. La fertilisation améliore nettement la situation, bien que sur le plan chimique, le sol soit relativement bien pourvu. Les formules combinant $\mathrm{N}$, P et $\mathrm{K}$ sont de loin les plus favorables, ce qui confirme bon nombre d'observations antérieures (GIULImond, 1965).

Afin de mieux comprendre ces difficultés de nutrition, nous avons procédé à des observations annexes portant d'abord sur les racines.

\section{1. - Observations sur l'enracinement}

Des mensurations ont été réalisées sur les racines d'une dizaine de plants ( $T$ et NPK) après dégagement soigné du système radiculaire. Les chiffres portés au tableau 8 illustrent de façon éloquente l'extrême faiblesse du développement racinaire du peuplier sur un sol argileux non structuré. On admet, en effet, que la croissance des racines de peuplier est normalement de l'ordre de 1 à $1,5 \mathrm{~m} / \mathrm{an}$, au cours des premières années de plantation sur un sol à propriétés physiques favorables (POURTET).

TABLEAU 8

Observations sur l'enracinement

\begin{tabular}{|c|c|c|c|c|}
\hline & & & $T$ & NPK \\
\hline$d$ & 1967 & $\ldots \ldots \ldots \ldots \ldots$ & 30,5 & - \\
\hline$d$ & 1968 & $\ldots \ldots \ldots \ldots \ldots \ldots$ & 51,5 & 66,5 \\
\hline$l$ & 1967 & $\ldots \ldots \ldots \ldots \ldots \ldots \ldots$ & 33 & - \\
\hline & 1968 & $\ldots \ldots \ldots \ldots \ldots \ldots \ldots$ & 59 & 69,5 \\
\hline
\end{tabular}

$d$ : Rayon moyen d'enracinement, en fin d'année de végétation $(\mathrm{cm})$.

$l$ : Longueur moyenne des racines primaires superficielles en fin d'année de végétation $(\mathrm{cm})$.

Il est probable que la faiblesse de l'enracinement explique une bonne part des difficultés d'installation des plants sur la station.

La fertilisation semble, par ailleurs, jouer sur la croissance des racines. Si ses effets sur l'élongation sont faibles, son action sur la morphologie des racines est loin d'être négligeable: la fumure NPK favorise, en effet, le développement de nombreuses racines secondaires et augmente le diamètre des racines primaires.

(1) L'alimentation en eau sur cette station peut être considérée comme optimum. Le plan d'eau assure en effet un approvisionnement quasi-illimité. 


\section{2. - Difficultés de nutrition minérale}

Ces difficultés pourraient être en partie la conséquence du défaut d'enracinement que nous venons de signaler sur un sol dont la richesse chimique, notamment, en $\mathrm{N}$ total et $\mathrm{K}$ échangeable, suffirait pourtant théoriquement à couvrir les besoins nutritionnels de peupliers bien enracinés (Leroy, 1969). Les carences ont d'ailleurs tendance à disparaître en deuxième année quand l'enracinement a pris un peu d'ampleur (cf. tableau 5).

Ainsi, dans les conditions de l'essai, les plants ne semblent pas trouver suffisamment d'éléments minéraux dans le faible volume de terre qu'ils parviennent à utiliser. La fertilisation, en localisant une quanticé supplémentaire d'éléments à proximité des racines, rétablit une situation plus favorable à ı nutricion et à la croissance.

Il faut, cependant, remarquer quau départ et quelles que soient les condicions de milieu, les plants de peupliers ne disposent jamais de racines très développées; dans certains cas, les racines sont même supprimées avant la plantation. Ce dernier point laisserait supposer que les difficultés de nutrition rencontrées dans l'essai ne s'expliqueraient pas exclusivement par un mauvais enracinement. Nous allons passer en revue ci-dessous les quelques défauts dans les propriétés du sol qui peuvent venir accentuer les effets du manque d'enracinement.

Les hautes teneurs en argile et matière organique alliées à la présence de calcaire actif pourraient tout d'abord provoquer dans une certaine mesure des difficultés d'approvisionnement en $K$, en dépit des stocks élevés du sol en cet élément (SchachischaBEL, 1966). Un essai, conduit sur une pépinière riche en argile, calcaire actif et potassium où se manifestent des carences en $\mathrm{K}$, tendrait à confirmer cette hypothèse (LeRoY, non publié). A ces problèmes d'approvisionnement en $\mathrm{K}$, pourraient s'ajouter des difficultés de nutrition en $\mathrm{P}_{.2} \mathrm{O}_{5}$; les teneurs du sol en $\mathrm{P}_{2 .} \mathrm{O}_{5}$ assimilable sont, en effet, relativement faibles.

Pour l'azote, nous avons procédé à un test de minéralisation par incubation (1) afin d'avoir une idée sur la disponibilité du stock d'azote total présent dans le sol (ZöetTl, 1965). Ce stock est énorme et dépasse en moyenne 40 tonnes/ha pour les 30 premiers $\mathrm{cm}$. L'incubation a permis de calculer un taux de minéralisation de 1,42\% conduisant exclusivement à la formation d'azote nitrique. Ce résultat concorde avec les chiffres cités dans la littérature pour des sols à propriétés voisines de celui que nous avons étudié (Foguelman, 1966; Libors, 1968). Ce taux de 1,42\% est faible pour un sol à $\mathrm{C} / \mathrm{N}$ d'environ 12 , d'autant plus faible que l'incubation maximise la minéralisation. Ce résultat s'explique certainement par l'extrême stabilité des humus calciques (Duchaufour, 1965). Ainsi une minéralisation peu active, alliée au manque d'enracinement, serait à l'origine des difficultés de nutrition azotée. En admettant, en effet, une densité apparente de 1,3 , un rayon d'enracinement de $30 \mathrm{~cm}$ sur $40 \mathrm{~cm}$ de profondeur et une teneur en $\mathrm{N}$ total de $0,9 \%$, la quantité d'azote minéral mise à la disposition des racines au cours de la première année de végétation ne dépasse pas 17 à $18 \mathrm{~g} /$ plant, soit une quantité du même ordre de grandeur que celle apportée par la fertilisation. Cette dernière doublerait donc les disponibilités réelles en azote des

(1) Incubation en étuve à $28^{\circ}$ pendant six semaines. Taux d'humidité : capacité de rétenion. Incubation sur horizon $A_{1}$ correspondant au profil 2 (tableau 1). 
peupliers pendant leur phase d'installation. Il est possible, d'autre part, que l'azote nitrique apporté par l'ammonitrate favorise un excès de minéralisation de l'azote dans le sol en place (Libois, 1968).

\section{3. - Conclusion partielle}

La discussion des résultats conduit à attribuer les difficultés de nutrition et de croissance des plants essentiellement à la faiblesse du développement racinaire des plants. Trois facteurs viendraient aggraver ces difficultés : actif,

- un manque de mobilité du potassium en présence d'argile et de calcaire

- la pauvreté relative du sol en phosphore assimilable,

- un défaut de minéralisation du stock d'azote.

Nous verrons pourtant dans le chapitre suivant qu'un quatrième facteur vient s'ajouter à cette liste.

\section{V. - ACTION DE CONCURRENCE EXERCEE PAR LA VEGETATION HERBACEE}

Les premières observations nous avaient conduits en automne 1967 à envisager un certain nombre d'hypothèses pour expliquer la faiblesse des accroissements de première année. Parmi celles-ci figuraient les éventuels effets nuisibles que la flore herbacée pouvait exercer en deux domaine différents, sur la croissance du peuplier :

- concurrence pour la nutrition minérale,

- concurrence au niveau du développement racinaire. L'abondant feutrage des racines de plantes herbacées pouvait, en effet, accentuer les difficultés d'enracinement du peuplier sur un sol à propriétés physiques défavorables.

Les populiculteurs réduisent en général la concurrence par des façons culturales avant et après plantation. Cependant, sur un sol de marais, ces façons sont difficilement réalisables et ont même parfois un effet dépressif sur la croissance des plants, à la suite d'une destruction d'une partie de leur système radiculaire essentiellement concentré en surface (LEROY, 1969).

Afin d'éclairer ces problèmes de concurrence sur sol de marais (sol humique à gley), un nouveau dispositif en blocs fut installé sur la même station en novembre 1967 '1). Il rassemble quatre traitements répétés quatre fois :

$\mathrm{T}$ : Témoin. Ni engrais, ni fauchage,

$\mathrm{F}$ : la surface des placeaux est régulièrement fauchée au cours de la saison de végétation afin de détruire la flore herbacée ou réduire sa vigueur,

NP: la surface des placeaux est fauchée et les plants reçoivent des engrais azotés,

$\mathrm{N}$ : apport d'engrais azotés seulement.

(1) Modalités de plantation et de fertilisation en II, 2.2 à 2.4. Un travail du sol localisé au pied des plants, la première année de végétation, aurait pu être introduit dans ce dispositif. Ce ıype de traitement sera éventuellement inclus dans de nouveaux essais. 
Les premières observations conduisent à un faisceau de résultats très significatif (cf. tableau 9):

- la faiblesse des accroissements de première année est confirmée. Le traitement NF apporte un gain relatif de croissance du même ordre de grandeur que le traitement NPK (cf. Tableau 3),

\section{TABLEAU 9}

Résultats des observations réalisées en 1968 sur le dispositif destiné à mettre en évidence les effets de la concurrence herbacée

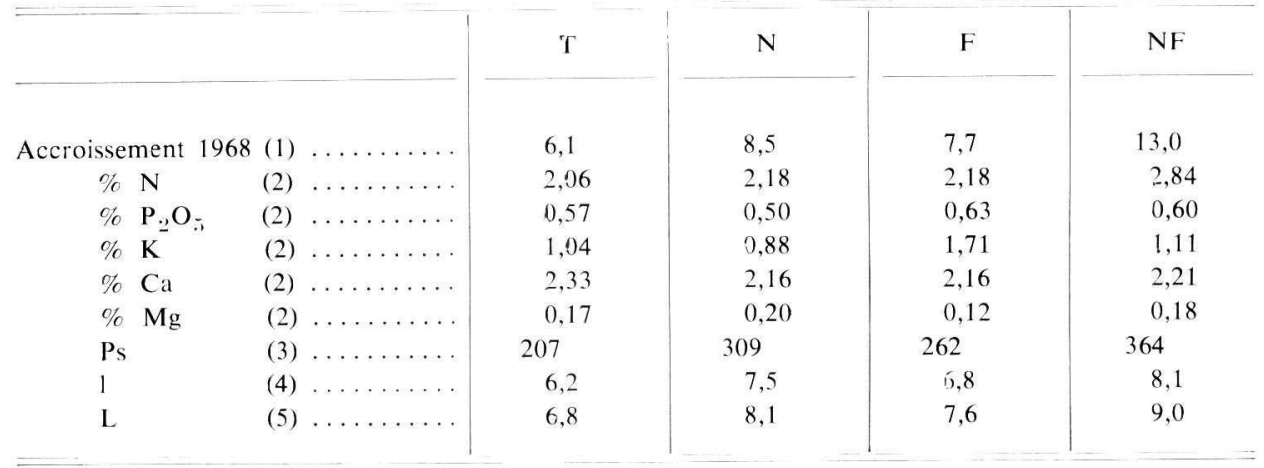

(1) Accroissement en circonférence en première année (nım).

(2) \% N... \% Mg: Taux des feuilles en éléments, exprimé par rapport à la matière sèche.

(3) Ps : Poids de 1.000 feuilles sèches (g).

(4) 1: Largeur des limbes en $\mathrm{cm}$.

(5) L : Longueur des limbes en $\mathrm{cm}$.

- des carences en $\mathrm{N}$ et $\mathrm{K}$ se manifestent sur les témoins et illustrent les difficultés de nutrition,

- le seul fauchage de la flore herbacée fait disparaître la carence en $\mathrm{K}$ et améliore la nutrition azotée, en $\mathrm{K}$,

- l'apport d'azote seul améliore la nutrition azotée, mais intensifie la carence

- le fauchage combiné à l'apport d'azote améliore nettement la nutrition, mais laisse persister une faible carence en potasse probablement liée à un effet de dilution,

- les effets de dilution qui interfèrent dans les analyses foliaires doivent être rapprochées des gains significatifs acquis grâce aux traitements sur les poids secs de 1.000 feuilles,

— sur le plan des poids et dimensions de feuilles, les traitements $\mathrm{N}$ et $\mathrm{F}$ diffèrent statistiquement à la fois du traitement $T$ et du traitement NF.

Cet ensemble de constatations vient compléter les conclusions partielles posées plus haut. En premier lieu, l'action défavorable de la végétation herbacée est clairement démontrée; elle porte sur la nutrition minérale avec des conséquences graves essentiellement pour $\mathrm{N}$ et $\mathrm{K}$. Remarquons au passage que la nutrition en ces deux éléments était déjà précaire par le seul fait des propriétés chimiques intrinsèques du sol. 
Le fauchage a également permis un gain de $23 \%$ sur la croissance en longueur des racines du peuplier. Cette action bénéfique sur les racines doit expliquer en partie les gains acquis grâce au fauchage.

Un nouvel élément d'information est enfin apporté par les analyses foiaires: les apports d'azote seul déséquilibrent profondément la nutrition phospho-potassique, ce qui confirme une fois de plus l'intérêt des formules combinées NPK.

\section{VI. - CONCLUSIONS}

\section{1. - Conclusions techniques}

Les essais sont installés sur un sol à gley de la Meuse, caractérisé par un anmoor caicique, une texture argileuse et une structure très compacte. Sur cette station chimiquement assez riche et bien alimentée en eau, la croissance des jeunes plants de peupiier s'avère extrêmement faible (accroissement annuel moyen des deux premières années sur la circonférence : 9,9 mm en l'absence de fertilisation). Dans ces conditions, les attaques de Dothichiza populea, parasite de faiblesse, conduisent à une mortaiité de $38 \%$.

Un défaut de développement radiculaire semble être à l'origine de cette situation. Les effets de ce défaut d'enracinement sont renforcés par une faible minéralisation de l'azote, des difficultés d'absorption du potassium et, enfin, par la concurrence herbacée. Ces phénomènes s'étendent à l'ensemble des peupleraies installées dans l'Est sur des stations analogues à celie où se déroulent les essais.

Sur ces stations, deux catégories de moyens d'intervention peuvent améliorer les conditions de croissance et permettre l'installation de peupleraies :

- l'élimination de la concurrence herbacée,

- la fertilisation.

Le travail du sol, généralement utilisé pour détruire la végétation herbacée, est pratiquement irréalisable sur sols à gley (sols de marais) ò̀ la portance est insuffisante. Les essais portant sur la destruction par fauchage des parties aériennes de la végétation herbacée ont montré l'intérêt de cette technique, quand elle est accompagnée de fertilisation. Une expérimentation nouvelle est envisagée dans ce domaine pour tester des moyens de destruction chimique avec la coilaboration de la Station de Syiviculture du C.N.R.F.

La fertilisation, à laquelle nous nous sommes davantage intéressés dans nos essais, apparaît comme très efficace. La formule NPK est de loin la plus favorable, tant à la nutrition qu'à la croissance et la résistance au Dothichiza:

- elle fait disparaître les carences,

- augmente la croissance des deux premières années de $77 \%$,

- abaisse le taux de mortalité de 38 à $13 \%$.

- favorise le développement radiculaire.

Les formules binaires NP et NK ont également des effets positifs sur la croissance, mais elles tendent à provoquer des déséquilibres nutritionnels. La formule PK enfin 
accentue les carences en azote, semble avoir un effet dépressif sur la croissance et augmente le taux de mortalité. Pour résumer les effets des engrais sur la croissance des plants, on pourrait proposer le classement suivant :

$$
\text { PK - T - P - K - N - (NP - NK) - NPK }
$$

Sur le plan pratique, ces résultats permettent dans l'immédiat de proposer une formule de fertilisation pour les sols de marais à anmoor calcique. Une combinaison NPK est conseillée :

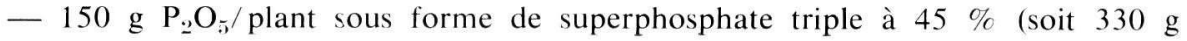
d'engrais environ). Apport en une fois au trou de plantation, en mélange avec la terre de remplissage, en prenant la précaution d'isoler les racines par une couche de terre non enrichie,

- $75 \mathrm{~g} \mathrm{~K}_{2} \mathrm{O}$ /plant sous forme de sulfate de potassium (soit environ $150 \mathrm{~g}$ d'engrais). Même mode d'apport que pour $\mathrm{P}_{2} \mathrm{O}_{5}$.

- Azote :

Juin $1^{\mathrm{ru}}$ année $\ldots 20 \mathrm{~g} \mathrm{~N} /$ plant $(60 \mathrm{~g}$ d'ammonitrate à $34,5 \%$, rayon d'épandage : $35 \mathrm{~cm}$ ),

Juin $2^{\circ}$ année $\ldots 60 \mathrm{~g} \mathrm{~N} /$ plant (180 g d'ammonitrate à $34,5 \%$, rayon d'épandage : $60 \mathrm{~cm}$ ).

Des doses d'engrais plus fortes ne présentent aucun intérêt, ainsi que l'a démontré un essai annexe dont nous n'avons pas jugé utile de publier les résultats.

La rentabilité immédiate de la formule de fertilisation proposée est très bien illustrée par le bilan des gains acquis grâce au seul effet positif des engrais sur la résistance au Dothichiza (chap. III et 3.3). Ce bilan laisse apparaître une économie de l'ordre de 350 à $400 \mathrm{~F} /$ ha sur les seuls regarnis.

\section{2. - Aspects méthodologiques}

Les essais poursuivis sur peuplier dans la Meuse montrent que les expérimentations de terrain peuvent conduire dans certains cas à des résultats rapidement utilisables.

La combinaison de dispositifs en blocs et de dispositifs «monoarbre»a, en effet, permis de tester un nombre important de traitements sans difficulté majeure. Les effets de ces traitements ont été contrôlés par diverses iechniques:

- mensurations portant sur la croissance des tiges,

- étude des caractéristiques dimensionnelles et pondérales des feuilles,

- analyses foliaires,

- observations sur les racines.

L'ensemble de ces techniques a conduit à un faisceau d'indications sur lesquelles des conclusions se sont rapidement fondées. Remarquons au passage que ces conclusions auraient été impossibles si le contrôle n'avait porté que sur les mensurations de tiges. 
Par ailleurs, la mise en place de dispositifs annexes a permis de vérifier les hypothèses fondées sur les résultats des premières observations; cette souplesse d'utilisation des dispositifs s'est avérée féconde.

Reçu pour publication en mars 1969.

\section{REMERCIEMENTS}

Nous tenons à remercier :

- M. l'Ingénieur en chef du G.R.E.F. Guédet, Chef du Centre de Gestion de l'O.N.F. à Verdun, pour sa collaboration,

- M. l'Agent Technique Claveau, en service à l'O.N.F., qui s'est chargé de la surveillance des essais et d'un grand nombre d'observations, statistiques.

- les services de Biométrie de l'I.N.R.A. qui ont effectués une partie des calculs

\section{SUMMARY}

RESULTS OF TRIALS OF POPLAR FERTILISATION ON GLEY SOILS OF THE MEUSE VALLEY.

The trials are standing on loamy calcareous gley soils of the Meuse valley, near Verdun. The humus are anmoor. On these soils of good chemical fertility (tab. I), the growth of young poplars appears very feeble (tab. 2 et 3 ). With those bad growth conditions, the fungal attacks of Dothichiza populea, a weakness parasite, give a $38 \%$ dying off, two years after plantation.

A lack of root extension (tab. 8) seems to be the main reason of this bad situation. The effects of that lack of root extension are strengthened by a feeble nitrogen mineralisation, difficulties of potassium nutrition and by the root competition of weeds.

Two kinds of technics may improve the growth conditions:

- the destruction of weeds

- the fertilization.

On gley soils, often too moist to permit the use of powerfull machines, the destruction of weeds may be realized by the mowing or the chemical phytocids. The mowing is appeared efficient in the trials; it has improved the nutrition and increased the growth (tab. 9).

The fertilization also gives good results; the $\mathrm{N}, \mathrm{P}, \mathrm{K}$ association is the best:

- it improves the nutrition (tab. 5)

- it increases the mean growth $(77 \%$ - tab. 3)

- it decreases the dying off rate (from 38 to $13 \%$ )

- it heips the root growth.

These results allow to give fertilization indications for the calcareous gley soils of the Eastern part of France where the poplars have growth difficulties. This fertilization has to be localized in the plantation hole (Chap. II, $\$ 23$ ). Besides, the manure must be accompanied by the destruction of weeds.

On a methudologic point of view, the trials has showed that a well led experimentation and a good control permit to obtain the first results in a short time (two years).

\section{ZUSAMMENFASSUNG}

ERSTE VORLAUFIGE ERGEBNISSE EINES PAPPELDÜNGEVERSUCHES AUF FINEM GLEYSTANDORT IM DEPARTMENT MEUSE.

Der Versuch ist auf einem Gleystandort angelegt (Bodenart: Ton, Humus : kalkiges Anmoor). Obwohl der Standort als gut nährstoff- und wasserversorgt angesehen werden kann 
(Tab. 1) zeigen jedoch die Pappeln ein äusserst geringes Wachstum (Tab. 2 und 3) und werden daher häufig von Dotichiza populea befallen (38\% Ausfall).

Das geringe Wachstum dürfte auf eine mangelhafte Entwicklung des Wurzelsystems zurück zuführen sein, deren Auswirkung noch durch eine geringe Mineralisation des Stickstoffes, eine unzureichende Kaliaufnahme und letztlich durch die Konkurrenz der Bodenvegetation verstärkt wird.

Es bestehen zwei Möglichkeiten um die Wachstumsbedingungen zu verbessern :

- Auschaltung der Unkrautkonkurrenz

- Düngung.

Der Einsatz von Traktoren ist auf nassen Gleyböden nur beschränkt möglich. Die konkurrenz der Bodenvegeiation kann jedoch durch Herbizide oder Mahd ausgeschaltet werden. Das Abmähen hat sich in diesem Versuch als sehr wirksam erwiesen und die Nährstoffaufnahme und das Wachstum der Pappeln verbessert (Tab. 9).

Die Düngung hat gleichfalls sehr gut Ergebnisse gezeigt, insbesondere eine Volldüngung (NPK) erwies sich als sehr vorteilhaft; die Nährstoffkarenzen varschwanden (Tab. 5), der Stärkenzuwachs erhöhte sich im Mittel um $77 \%$, der Ausfall wurde von $38 \%$ auf $13 \%$ verringert und die Wurzelentwicklung gefördert.

Die vorlieganden Ergebnisse gestatten der Praxis Hinweise für die Düngung von kalkreichen Gleystandorten in Ostfrankreich zu geben, auf welchen wir Zuwachshemmungen der Pappeln feststellen konnten. Es handeli sich dabei um eine Pflanzlochdüngung (Siehe Abschnitt II-23). Desweiteren wird vorgeschlagen durch Mahd oder Herbizide die Konkurrenz der Bodenvegetation auszuschalten.

Bezüglich der Versuchsmethodik konnte gezeigt werden, dass eine entsprechende Versuchsanordnung und eine sorgfältige Kontrolle sehr rasch (- bereits nach Jahren -) zu hrauchbaren Ergebnissen führen kann.

\section{REFERENCES BIBLIOGRAPHIQUES}

Chardenon J., 1957. L'empioi des fertilisants en populiculture. Actes VIr Congr. internation. Peuplier, Direction des Forêts, 313-318.

Contant P., 1957. Essai de fumure minérale d'une plantation de peupliers. Actes VI Congr. internation. Peuplier. Direction des Forêts, Paris, 319-324

Duchaufour Ph., 1965. Précis de Pédologie, Masson, Paris.

Foguelman R., 1966. Etude de l'activité biolog:que, en particulier de la minéralisation de l'azote. Doc. $n^{\circ} 30$, Centre Et. phytosociol. écol., Montpellier.

Giulimond G., 1965. Investigations sur la nutrition minéralı du peuplier par le moyen des analyses foliaires. Variations de teneurs en cendres sur des essais de fertilisation. Publ. Centr. Sper. Agr. For., 8, 41-54.

LERoy Ph., 1969. La fertilisation du peuplier: connaissances acquises et difficultés d'application Rev. forest. fr., 3, 163-182.

Libois A., 1968. Dynamique de l'azote minéral en sol nu. Ann. agr., 19 (2), 103-128.

Millier C., 1967. Comparaisons multiples de moyennes: Test de Duncan, Document I.N.R.A., Station de Biométrie, 54 - Champenoux.

Pourtet J., 1957. La culture du peuplier, Baillière et fils, Paris.

Schachtschabel P., Scheffer F., 1966. Bodenkunde, Stuttgart, F. Enke,

TARIS B., 1957. Contribution à l'étude des maladies cryptogamiques des rameaux et des jeunes plants de peupliers. Thèse, Alençon.

Van der Meiden H. A., Kolster H. W., 1964. Variations in the mineral content of poplar leaves during the vegetation season and in different parts of the plant. Ned. Bosbouw Tijdschr., 36 (1), 1-11.

ZôEttL H., 1965. Anhäufung und Umsetzung von Stickstoff im Waldboden. Deut. Bot. Gesellsch, 78 (4), 167-180. 


\section{NOTE DE L'AUTEUR}

Les difficultés d'installation du peuplier sur sols à gley calciques ont pu être attribuées à un ensemble de faiıs défavorables à la vie des plants, mais propices au développement des champignons parasites. La fertilisation et la destruction des adventices sur des plantations réalisées au printemps, pourraient en partie pallier ces difficultés.

Des études complémentaires nous ont cependant permis de remarquer que les clônes couramment utilisés semblaient mal adaptés au climat lorrain. Cette constatation jointe à l'apparition du Marssonina Populea dans l'est de la France, engage à la prudence. Dans ces conditions, les conclusions de l'article, tout en restant valables sur le fond doivent être nuancées par des considérations climatiques et génétiques. Des recherches nouvelles devraient ềre engagées sur le choix de clônes susceptibles de mieux supporter le climat lorrain que les c'ônes traditionnels. 